\title{
Actualidades en el manejo sistémico del cáncer de mama
}

\author{
Current affairs in breast cancer systemic treatment \\ Rosa Luz Luna-Palencia, ${ }^{*}$ Eliseo Neftali De la Cruz-Escobar ${ }^{\ddagger}$
}

\begin{abstract}
* Oncóloga Médica, Departamento de Oncología Médica del Hospital Ángeles Lindavista. Ciudad de México, México.

‡ Oncólogo Médico, adscrito al Departamento de Oncología Médica del Hospital de Gineco-Obstetricia No. 3, Unidad Médica de Alta Especialidad, Centro Médico Nacional La Raza, Instituto Mexicano del Seguro Social. Ciudad de México, México.
\end{abstract}

Correspondencia: Rosa Luz Luna-Palencia Riobamba Núm. 639, Lindavista Sur, 07760, Alcaldía Gustavo A. Madero, Ciudad de México. E-mail: rlpalencia.oncologia@gmail. com

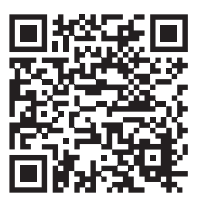

\section{RESUMEN}

El cáncer de mama representa la principal causa de muerte por cáncer en mujeres. Se distinguen cuatro fenotipos moleculares asociados a un distinto pronóstico y tratamiento. En el manejo del cáncer de mama con receptores hormonales positivos y sin sobreexpresión de HER2 el principal avance se ha visto en el contexto metastásico al agregar inhibidores de cinasas dependientes de ciclinas $4 / 6$ al tratamiento endocrino, obteniendo medianas de supervivencia libre de progresión de 27.6 meses con palbociclib/letrozol (PALOMA-2) o de supervivencia global de 58.7 meses al agregar ribociclib al tratamiento hormonal en mujeres pre- o perimenopáusicas (MONALEESA-7). Las terapias anti-HER2 han mejorado de forma considerable el pronóstico de las pacientes con cáncer de mama con sobreexpresión del oncogén HER2, en el contexto de enfermedad temprana mediante la adyuvancia con trastuzumab o trastuzumab emtansina y en contexto metastásico agregando pertuzumab al tratamiento con trastuzumab y docetaxel (CLEOPATRA) con medianas de supervivencia global de 56.5 meses con el doble bloqueo. En el subgrupo de pacientes triple negativas, la adición de inmunoterapia con el inhibidor de PD-L1 (ligando 1 de muerte programada [en inglés: Programmed Death-Ligand 1]) atezolizumab a la quimioterapia con nab-paclitaxel (IMpassion 130) demostró una mediana de supervivencia global de 25 meses en pacientes con expresión positiva de PD-L1.

Palabras clave: Cáncer de mama, inhibidores de cinasas dependientes de ciclinas, sobreexpresión de HER2, trastuzumab emtansina, PD-L1, inmunoterapia.

\section{ABSTRACT}

Breast cancer is the leading cause of dead because of cancer in women. Four molecular phenotypes are known, associated with a distinct prognosis and treatment options. In the treatment of breast cancer with positive hormonal receptors, without overexpression of HER2, the major progress have been seen in metastatic setting with the addition of cyclin dependent kinases 4/6 inhibitors to endocrine therapy, with median progression-free survival of 27.6 months with palbociclib/letrozole (PALOMA-2 trial) or overall survival of 58.7 months with the addition of ribociclib to the hormonal therapy in premenopausal women (MONALEESA-7 trial). Anti-HER2 therapies have improved considerably the prognosis of the patients with breast cancer with overexpression of HER2, in early breast cancer with adjuvant trastuzumab or trastuzumab emtansine and in metastatic setting with the addition of pertuzumab to trastuzumab and docetaxel therapy (CLEOPATRA trial) with median overall survival of 56.5 meses with the double block. In triple negative women, the addition of immunotherapy with the PD-L1 inhibitor atezolizumab to the chemotherapy with nab-paclitaxel (IMpassion 130 trial) showed a median overall survival of 25 months in patients with positive expression of PD-L1.

Keywords: Breast cancer, cyclin dependent kinases inhibitors, HER2 overexpression, trastuzumab emtansine, PD-L1, immunotherapy.

Citar como: Luna-Palencia RL, De la Cruz-Escobar EN. Actualidades en el manejo sistémico del cáncer de mama. Rev Mex Mastol. 2020; 10 (3): 71-82. https://dx.doi.org/10.35366/99156 


\section{INTRODUCCIÓN}

A nivel mundial el cáncer de mama representa la neoplasia maligna diagnosticada con mayor frecuencia y la principal causa de muerte por cáncer en mujeres. ${ }^{1}$

Mediante el análisis de expresión genómica se han encontrado cuatro fenotipos moleculares del cáncer de mama y estos perfiles de expresión genómica pueden aproximarse mediante técnicas accesibles como el uso de anticuerpos diagnósticos y la valoración de la morfología tumoral. La inmunohistoquímica del receptor de estrógeno, del receptor de progesterona, el análisis del índice de proliferación Ki-67 y la sobreexpresión del receptor 2 del factor de crecimiento epidérmico (HER2) se pueden utilizar como marcadores subrogados para aproximar una clasificación molecular. A partir de esto, el cáncer de mama puede clasificarse como luminal A (con fuerte expresión de receptores hormonales, sin sobreexpresión de HER2 y bajos índices de proliferación), luminal B (con expresión moderada a débil de receptores hormonales, índices de proliferación altos, grado histológico 3, pueden contar o no con sobreexpresión de HER2), triple negativo (sin expresión de receptores hormonales, sin sobreexpresión de HER2) y HER2 puro (sin expresión de receptores hormonales, con sobreexpresión de HER2). ${ }^{2}$ Es importante reconocer estos diferentes subtipos moleculares, no sólo por sus diferencias intrínsecas en pronóstico, supervivencia o patrones de recurrencia, sino también, y de vital importancia, por las diferentes opciones de tratamiento que conlleva cada una de estos subtipos.

En la población mexicana $60 \%$ de las pacientes con cáncer de mama tendrá receptores hormonales positivos, $20.4 \%$ tendrá sobreexpresión de HER2 y $23.1 \%$ tendrá fenotipo triple negativo. ${ }^{3}$

ACTUALIDADES EN EL MANEJO DEL CÁNCER DE MAMA CON RECEPTORES HORMONALES POSITIVOS (RH+)/HER2 NEGATIVO

Más de 70\% de las pacientes con cáncer de mama poseen receptores hormonales positivos y HER2 negativo. ${ }^{4}$ En este subgrupo de pacientes se cuenta con ensayos clínicos aleatorizados en los que se compara el efecto de la quimioterapia como tratamiento de primera línea en contraposición con tratamiento hormonal de primera línea, los cuales han demostrado que el tratamiento inicial con quimioterapia está asociado con mayores tasas de respuesta; sin embargo, sin mejoría en la supervivencia global (SG) y con mayor toxicidad asociada con la quimioterapia. ${ }^{5}$ Por tanto, la terapia endocrina es considerada la primera línea de tratamiento en pacientes cuyo cáncer de mama presenta receptores hormonales positivos, excepto en aquellos casos con crisis visceral, definida por disfunción orgánica severa y progresión rápida de la enfermedad, en la cual se requiere de una terapia sistémica más rápida y con mayor tasa de respuesta. ${ }^{6}$

La expresión y activación del receptor estrogénico es importante para el control del crecimiento tumoral, por tanto, los fármacos que bloquean selectivamente el receptor estrogénico (antagonistas como fulvestrant o moduladores como tamoxifeno) o aquellos que bloquean la biosíntesis de estrógenos (inhibidores de aromatasa) representaron durante años la terapia inicial en el tratamiento del cáncer de mama metastásico con RH+/HER2 negativo sin crisis visceral. ${ }^{7}$ Ensayos clínicos aleatorizados en mujeres postmenopáusicas con cáncer de mama avanzado, comparando la terapia endocrina con inhibidores de aromatasa (primera, segunda y tercera generación) en contraposición con otra terapia endocrina (tamoxifeno, acetato de megestrol, acetato de medroxiprogesterona y fulvestrant), muestran un beneficio en supervivencia global con el uso de inhibidores de aromatasa (HR 0.90, 95\% IC 0.84-0.97, $p=0.007) .{ }^{8}$

De uso habitual en la práctica clínica, los inhibidores de aromatasa de tercera generación (anastrozol, letrozol y exemestano) comparados con tamoxifeno, brindan un beneficio al menos en supervivencia libre de progresión (SLP) y respuesta. La comparación directa en un estudio fase III aleatorizado de letrozol vs tamoxifeno arrojó una supervivencia libre de progresión de 9.4 meses con letrozol en comparación con seis meses con tamoxifeno ( $p$ < 0.0001) y una tasa de respuesta de $32 \%$ con letrozol y de $21 \%$ con tamoxifeno $(p=0.0002) .{ }^{9}$ La misma comparación utilizando exemestano arrojó una mediana de supervivencia libre de progresión de 9.9 meses con exemestano en comparación 
con 5.8 meses con tamoxifeno $(p=0.028)$, con una tasa de respuesta de $46 \%$ en comparación con $31 \%$, respectivamente $(p=0.005) .{ }^{10}$

La aromatasa cataliza la conversión de androstenediona, testosterona y $16 \alpha$-hidroxitestosterona a estrona (E1), 17 $\beta$-estradiol (E2) y $17 \beta, 16 \alpha$-estriol, de manera respectiva. Desde el descubrimiento del inhibidor de aromatasa de primera generación aminoglutetimida, los llamados inhibidores de tercera generación presentan alta selectividad por la aromatasa, por lo que recibieron la aprobación por la Food and Drug Administration (FDA) para su uso en el cáncer de mama con $\mathrm{RH}$ positivos a finales de los años $90 .{ }^{11}$ De acuerdo con su estructura química, los inhibidores de aromatasa se clasifican en dos subtipos: tipo I o esteroideos como el exemestano, los cuales se unen covalentemente al sitio activo de la enzima resultando en inhibición irreversible (inhibidores suicidas), y tipo II o no esteroideos como el anastrozol y letrozol, los cuales se unen de manera no covalente a la aromatasa y saturan su sitio activo, generando una inhibición reversible. ${ }^{12}$ Los inhibidores de aromatasa no esteroideos son derivados del imidazol en contraste con los esteroideos, los cuales son derivados de la androstenediona y poseen efectos agonistas androgénicos débiles. La importancia clínica de estas diferencias se refleja en la falta de resistencia cruzada entre los compuestos de ambos tipos, al menos cuando un inhibidor de aromatasa esteroideo es administrado en segunda línea posterior al tratamiento con un inhibidor de aromatasa no esteroideo. ${ }^{13}$ Existe también evidencia, aunque limitada, del beneficio en el tratamiento de segunda línea con letrozol en pacientes que progresaron a una primera línea de tratamiento con exemestano, conservando beneficio clínico de $55.6 \%$ con una mediana de tiempo a la progresión de 9.3 meses. ${ }^{14}$

Pese a que la terapia endocrina es una estrategia de tratamiento efectiva en el manejo inicial de las pacientes con cáncer de mama $\mathrm{RH}$ positivos/HER2 negativo metastásico, no todas las pacientes responderán a este esquema endocrino de primera línea (resistencia primaria), mientras que aquellas que respondieron inicialmente progresarán (resistencia secundaria). ${ }^{15}$ Una vía de señalización que resulta clave en el desarrollo del cáncer de mama luminal es la de fosfatidilinositol-3-cinasa (PI3K)/Akt/blanco de rapamicina en células de mamíferos (mTOR). El tratamiento neoadyuvante con letrozol reduce los marcadores de actividad de PI3K, lo cual se correlaciona con una mayor supervivencia libre de progresión $(p=0.02),{ }^{16}$ por tanto, es posible que la deprivación estrogénica disminuya el crecimiento tumoral en el cáncer de mama luminal en parte por la disminución en la actividad de esta vía de proliferación celular. Por otra parte, existe evidencia de que esta vía de señalización se ve activada durante la resistencia secundaria a la terapia endocrina, promoviendo la supervivencia tumoral pese al bloqueo estrogénico. La mayoría de las células de cáncer de mama que se adaptaron a la terapia endocrina aún expresan receptor estrogénico y sensibilidad estrogénica, por lo tanto, el tratamiento con inhibidores de PI3K sin inhibición estrogénica resultaría insuficiente para detener al máximo el crecimiento tumoral. ${ }^{17}$

Sobre la base de lo anterior, se ha probado en un ensayo clínico fase III (bolero 2) la eficacia de everolimus junto con exemestano en pacientes con cáncer de mama avanzado, con receptores hormonales positivos, con progresión o recurrencia al tratamiento con inhibidor de aromatasa no esteroideo. En dicho ensayo se aleatorizaron a 724 pacientes en relación 2:1 a tratamiento con everolimus (derivado de sirolimus que inhibe a mTOR mediante su unión alostérica a mTORC1) o placebo con exemestano, demostrando una mediana de supervivencia libre de progresión de 6.9 meses para everolimus con exemestano en contraposición a 2.8 meses para placebo con exemestano (HR para progresión o muerte 0.43 ; $95 \%$ IC 0.35 a $0.54 ; p<0.001)$ y tasa de respuesta de 9.5 vs $0.4 \%$, respectivamente $(p<0.001) .{ }^{18}$ Beneficios igual de modestos se han reportado en la aleatorización a everolimus más tamoxifeno en contraposición con tamoxifeno monodroga en pacientes con resistencia a inhibidor de aromatasa (TAMRAD), demostrando una disminución de $46 \%$ en el riesgo de progresión al agregar everolimus al tratamiento con tamoxifeno ( $\mathrm{HR}$ $0.54,95 \%$ IC 0.36 a 0.81 , mediana de tiempo a la progresión de 8.6 meses con tamoxifeno más everolimus en contraposición a 4.5 meses con tamoxifeno monodroga, $\mathrm{p}=0.002) .{ }^{19}$

Los mecanismos de activación de mTOR son complejos y parcialmente dependientes de PI3K, 
por tanto, se ha evaluado la eficacia de buparlisib (inhibidor de las cuatro isoformas de PI3K de clase I) o placebo combinado con fulvestrant en el tratamiento de las pacientes con cáncer de mama metastásico resistente a inhibidor de aromatasa, obteniendo una mejoría en supervivencia libre de progresión de aproximadamente dos meses al agregar buparlisib al tratamiento con fulvestrant (6.9 meses en contraposición a cinco meses con fulvestrant monodroga HR 0.78, 95\% IC 0.67 a 0.89 ). ${ }^{20}$ El beneficio clínico de la inhibición de la vía de PI3K o AKT en pacientes con resistencia a terapia endocrina resulta limitada, ya que esta inhibición produce una regulación a la alza o activación de diversos receptores de tirosina cinasa, cuya activación puede anular el efecto sobre la inhibición de PI3K. Por lo tanto, el tratamiento combinado de inhibidores de HER2 y PI3K, HER2 y AKT, HER2 y TORC1 o EGFR y AKT podrían ser de mayor beneficio clínico que el tratamiento monodroga. ${ }^{17}$

Aproximadamente $40 \%$ de las pacientes con cáncer de mama con receptores hormonales positivos y HER2 negativo poseen una mutación activadora en el gen PIK3CA, lo cual genera hiperactivación de la isoforma alfa de PI3K (p110 $\alpha$ ). Alpelisib es un inhibidor selectivo de $p 110 \alpha$, el cual probó su efectividad en el ensayo clínico fase III que comparó alpelisib más fulvestrant con placebo más fulvestrant en pacientes con resistencia endocrina, resultando en una supervivencia libre de progresión de 11 meses en el grupo de alpelisib más fulvestrant vs 5.7 meses en el grupo de placebo más fulvestrant (HR 0.65, 95\% IC 0.5 a 0.85 , $\mathrm{p}<0.001)$ y una tasa de respuesta de 26.6 vs $12.8 \%$, respectivamente. ${ }^{21}$

El ciclo celular es visto como una serie de fases que progresan de una manera ordenada (G1, S, G2, M) con varias combinaciones de cinasas dependientes de ciclinas (CDKs) regulando el paso de una fase a otra. Las CDKs pertenecen a una familia de proteínas cinasas de serina/treonina que incluye tres CDKs de interfase (CDK2, CDK4, CDK6), una CDK mitótica (CDK1), CDKs regulatorias (CDK7) y CDKs transcripcionales (CDK8, CDK9). Las ciclinas son una familia diversa de proteínas que se subdividen en cuatro clases (A-, B-, D-, E-) que participan como subunidades regulatorias en los complejos CDK/ciclinas. La represión del ciclo celular se mantiene mediante el secuestro de la familia de los factores de transcripción E2F por el producto del gen de retinoblastoma (pRB). En respuesta a factores mitogénicos, una célula quiescente sintetiza ciclina D1, la cual forma un complejo activador con CDK4/CDK6, iniciando así la fosforilación del pRB, el cual al estar fosforilado anula su efecto represor sobre E2F propiciando así la transcripción de genes específicos de la fase $S$. Uno de esos genes promueve la síntesis de ciclina $\mathrm{E}$, la cual se asocia con CDK2 y fosforila al pRB, llevando a la célula irreversiblemente de fase G1 a fase S. En la fase $\mathrm{S}, \mathrm{CDK} 2$ es también activada por la ciclina A2 haciendo posible la transición de la fase $\mathrm{S}$ a la fase G2 y finalmente CDK1 es activada por ciclinas A/B para facilitar la progresión a la mitosis. ${ }^{22}$ Por tanto, la actividad catalítica de CDK4 o CKD6 regula un punto crítico para la transición de la fase G1 a la fase $\mathrm{S}$ comprometiendo a la célula a su división. Más de 90\% de las células tumorales adquieren alguna alteración en los mecanismos de control de esta transición de fase, como regulación a la alza de CDK4, amplificación de las ciclinas D, regulación a la baja de p16 $6^{\mathrm{INK} 4 \mathrm{~A}}$ (inhibidor de CDK4), mutaciones en CDK4 que imposibilitan la unión de $\mathrm{p} 16^{\mathrm{INK} 4 \mathrm{~A}}$ a la enzima o mutaciones en retinoblastoma, todo esto generando pérdida del control proliferativo. ${ }^{23}$ En el cáncer de mama se han descrito alteraciones en diversas proteínas reguladoras del ciclo celular, encontrando una desregulación en el eje D1:CDK4/6, con el hallazgo de sobreexpresión de ciclina D1 en algunos tumores y la dependencia de CDK4 y ciclina D1 para la inducción de neoplasias malignas mamarias en ratones. ${ }^{23}$

En la actualidad, existen tres inhibidores de CDK4 y CDK6 aprobados para su uso en pacientes postmenopáusicas con cáncer de mama $\mathrm{RH}$ positivos/HER2 negativo en primera línea de tratamiento en combinación con un inhibidor de aromatasa: palbociclib, ribociclib y abemaciclib son inhibidores de CDK4 y CDK6 administrados vía oral; abemaciclib es el inhibidor más potente, tiene además efecto inhibitorio sobre CDK9 y ha mostrado tener habilidad para cruzar barrera hematoencefálica. ${ }^{24}$ Los resultados obtenidos con la inclusión de estos inhibidores de CDK4 y CDK6 representó una mejoría clínica y estadísticamente signifi- 
cativa en la supervivencia libre de progresión de hasta 27.6 meses con palbociclib/letrozol (95\% IC 22.4-30.3) en comparación con 14.5 meses con placebo/letrozol (95\% IC 12.3-17.1) (HR 0.563; $p<0.0001)$ en la actualización del estudio PALOMA-2 (Tabla 1). ${ }^{25,26}$ El inicio de la primera línea de quimioterapia se vio también retrasada con el uso de palbociclib/letrozol con una mediana de inicio a los 40.4 meses en comparación con los 29.9 meses en el grupo de pacientes tratado con placebo-letrozol. ${ }^{26} \mathrm{En}$ pacientes premenopáusicas o perimenopáusicas el estudio MONALEESA-7 analizó el efecto de agregar ribociclib a la terapia endocrina, sin permitir entrecruzamiento de grupos a la progresión, obteniendo un beneficio en cuanto a supervivencia libre de progresión comparable al mostrado en pacientes postmenopáusicas, observando además una mejoría en supervivencia global con la adición de ribociclib a la terapia endocrina, con $70.2 \%$ de las pacientes vivas a 42 meses en el grupo de ribociclib en comparación con el $46 \%$ de las pacientes que recibieron placebo (HR para muerte de 0.71 ; $95 \%$ IC, $0.54-0.95, p=0.00973),{ }^{30,31}$ una mediana de supervivencia global de 58.7 meses al agregar ribociclib al tratamiento hormonal, en comparación con 48 meses con placebo. En cuanto a toxicidad, palbociclib y ribociclib presentan principalmente toxicidad hematoló- gica, por el contrario, la toxicidad hematológica con abemaciclib es menos pronunciada, siendo su principal efecto adverso la diarrea. Con palbociclib se ha reportado neutropenia G3-4 en $54-66 \%$, leucopenia $19-25 \%$, anemia $2-6 \%$, en cuanto a ribociclib se agrega elevación G3-4 de transaminasas en 5-10\% de las pacientes, así como prolongación del intervalo QT en 1-3\%. La tasa de neutropenia febril es de 1-2\% con palbociclib y ribociclib y $<1 \%$ con abemaciclib. ${ }^{24}$

Los inhibidores de CDK4 y CDK6 se evaluaron también en progresión a primera línea de tratamiento endocrino, en esta ocasión junto con el antagonista del receptor estrogénico fulvestrant a la dosis que demostró mejores resultados en cuanto a supervivencia libre de progresión en monodroga. ${ }^{32}$ Los ensayos clínicos fase III han demostrado una mejoría en supervivencia libre de progresión de cinco a siete meses al agregar los inhibidores de CDK4 y CDK6 al tratamiento con fulvestrant en segunda línea de tratamiento (Tabla 2). En el grupo de pacientes que presentaron sensibilidad al tratamiento endocrino de primera línea (definida como haber presentado respuesta completa, respuesta parcial o enfermedad estable por $\geq$ 24 semanas con al menos una terapia endocrina recibida en enfermedad avanzada o haber recibido al menos 24 meses de terapia endocrina adyuvante previo a la progresión) se observó

\begin{tabular}{|c|c|c|c|c|}
\hline \multicolumn{5}{|c|}{$\begin{array}{l}\text { Tabla 1: Ensayos clínicos fase III, aleatorizados, doble ciego, con inhibidores } \\
\text { de CDK4/CDK6, en primera línea de tratamiento en mujeres con cáncer de mama } \\
\text { RH positivos/HER2 negativo avanzado. }\end{array}$} \\
\hline Autor (estudio) & Tratamiento & $\mathrm{n}$ & mSLP (meses) & Respuesta* \\
\hline $\begin{array}{l}\text { Finn RS, et al. } \\
(\text { PALOMA-2) }\end{array}$ & $\begin{array}{c}\text { Palbociclib + letrozol vs } \\
\text { placebo + letrozol }\end{array}$ & $666^{\ddagger}$ & $\begin{array}{c}27.6 \text { vs } 14.5 \\
(H R 0.563 ; p<0.0001)\end{array}$ & 55.3 vs $44.4 \%$ \\
\hline $\begin{array}{l}\text { Hortobagyi GN, et al. } \\
(\text { MONALEESA-2) })^{27,28}\end{array}$ & $\begin{array}{l}\text { Ribociclib + letrozol vs } \\
\text { placebo + letrozol }\end{array}$ & 668 & $\begin{array}{c}25.3 \text { vs } 16 \\
\left(\operatorname{HR} 0.58 ; p=9.63 \times 10^{-8}\right)\end{array}$ & 54.5 vs $38.8 \%$ \\
\hline $\begin{array}{l}\text { Goetz MP, et al. } \\
(\text { MONARCH 3) }\end{array}$ & $\begin{array}{l}\text { Abemaciclib + IANE vs } \\
\text { placebo + IANE }\end{array}$ & $C_{1=C}^{493}$ & $\begin{array}{l}\text { HR } 0.54 ; p=0.000021 \\
\text { (mSLP NA para abemaciclib, } \\
14.7 \text { con placebo) }\end{array}$ & 59.2 vs $43.8 \%$ \\
\hline $\begin{array}{l}\text { Tripathy D, et al. } \\
(\text { MONALEESA-7) }\end{array}$ & $\begin{array}{l}\text { Ribociclib vs placebo }+ \\
\text { tamoxifeno o IANE }\end{array}$ & 672 & $\begin{array}{c}23.8 \text { vs } 13 \\
(H R 0.55 ; p<0.0001)\end{array}$ & 51 vs $36 \%$ \\
\hline
\end{tabular}

* Pacientes con enfermedad medible. ${ }^{\ddagger}$ Aleatorización 2:1 palbociclib vs placebo. ${ }^{\S}$ Ensayo clínico fase III en pacientes pre- 0 perimenopáusicas. " Con goserelina mensual.

$\mathrm{RH}=$ Hazard ratio; HER2 = receptor del factor de crecimiento epidérmico humano 2; $\mathrm{mSLP}=$ mediana de supervivencia libre de progresión; IANE = inhibidor de aromatasa no esteroideo; NA = no alcanzada. 
Tabla 2: Ensayos clínicos fase III, aleatorizados, ${ }^{*}$ doble ciego, con inhibidores de CDK4/CDK6 y fulvestrant, ${ }^{\ddagger}$ en progresión a tratamiento endocrino de primera línea en mujeres con cáncer de mama RH positivos/HER2 negativo avanzado.

\begin{tabular}{|c|c|c|c|c|}
\hline Autor (estudio) & Tratamiento & $\mathrm{n}$ & mSLP (meses) & Respuesta§ \\
\hline $\begin{array}{l}\text { Cristofanilli M, et al. } \\
\text { (PALOMA-3) }^{33,34 \pi}\end{array}$ & $\begin{array}{c}\text { Palbociclib + fulvestrant } \\
\text { vs placebo + fulvestrant } \|\end{array}$ & 521 & $\begin{array}{c}9.5 \text { vs } 4.6 \\
\text { (HR } 0.46 ; p<0.0001)\end{array}$ & $\begin{array}{c}24.6 \text { vs } 10.9 \% \\
(p=0.0012)\end{array}$ \\
\hline $\begin{array}{l}\text { Slamon D, et al. } \\
(\text { MONALEESA-3) } \\
\text { (M5** }\end{array}$ & $\begin{array}{l}\text { Ribociclib + fulvestrant } \\
\text { vs placebo + fulvestrant }\end{array}$ & 726 & $\begin{array}{c}20.5 \text { vs } 12.8 \\
\text { (HR 0.593; } ; \text {; } p<0.001) \\
\text { (HR } 0.565 ; \S \S \\
\text { IC } 95 \% 0.428-0.744)\end{array}$ & $\begin{array}{c}40.9 \text { vs } 28.7 \% \\
(p=0.003)\end{array}$ \\
\hline $\begin{array}{l}\text { Sledge GW, et al. } \\
\left(^{\text {MONARCH 2) }}\right)^{36 \pi}\end{array}$ & $\begin{array}{l}\text { Abemaciclib + fulvestrant } \\
\text { vs placebo + fulvestrant } \|\end{array}$ & 669 & $\begin{array}{c}16.4 \text { vs } 9.3 \\
\text { (HR } 0.553 ; p<0.001)\end{array}$ & $\begin{array}{c}48.1 \text { vs } 21.3 \% \\
(p<0.001)\end{array}$ \\
\hline
\end{tabular}

* Aleatorización 2:1. ${ }^{\ddagger}$ Fulvestrant 500 mg vía intramuscular D1 y D15 del ciclo, después el D1 de cada ciclo de 28 días. $\S$ Pacientes con enfermedad medible. " Se admiten pacientes sin importar sus estatus de menopausia. "I Las pacientes pre- 0 perimenopáusicas reciben además goserelina durante la administración de fulvestrant. ** Se incluye pacientes vírgenes a tratamiento y con progresión a tratamiento endocrino. Sólo pacientes postmenopáusicas. \# Población global (primera y segunda línea de tratamiento). $\S^{\S} \mathrm{HR}$ en segunda línea de tratamiento.

$\mathrm{RH}=$ receptores hormonales; HER2 $=$ receptor del factor de crecimiento epidérmico humano 2; $\mathrm{mSLP}=$ mediana de supervivencia libre de progresión.

además mejoría en supervivencia global al agregar palbociclib al tratamiento endocrino, con una mediana de supervivencia global de 39.7 meses en el grupo que recibió palbociclib/ fulvestrant en contraste con 29.7 meses en el grupo aleatorizado a placebo/fulvestrant (HR $0.72 ; 95 \%$ IC $0.55-0.94) .{ }^{34}$

\section{ACTUALIDADES EN EL MANEJO DEL CÁNCER DE MAMA HER2 POSITIVO}

La sobreexpresión del oncogén HER2 (erbB-2/ neu) se encuentra en aproximadamente $15 \mathrm{a}$ $30 \%$ de las pacientes con cáncer de mama invasor y representa un marcador predictivo de respuesta a terapias específicas. Previo al desarrollo de estos tratamientos la sobreexpresión de este marcador era considerada como un factor de pobre pronóstico debido a que se relacionaba con un incremento de recurrencia y mortalidad; sin embargo, con el advenimiento de nuevas terapias el pronóstico ha mejorado de forma considerable.

El oncogén HER2 codifica para un receptor de membrana con actividad tirosinacinasa, el cual es un miembro de la familia de receptores del factor de crecimiento epidérmico (EGFR). La homodimerización o heterodimerización de este receptor con otros miembros de la familia de EGFR, generalmente asociada a su sobreexpresión, produce señales intracelulares corriente abajo que concluyen en la activación de genes de proliferación y carcinogénesis. ${ }^{37,38}$

Los métodos más utilizados para evaluar la expresión de HER2 son la inmunohistoquímica (IHQ) y la hibridación fluorescente in situ (FISH). Con la IHQ se evalúa el grado de expresión de la proteína en la membrana de las células tumorales y con FISH el número de copias del gen en el núcleo celular para detectar su amplificación. Los pacientes candidatos a recibir terapia anti-HER2 deben cumplir con alguna de estas dos condiciones: inmunohistoquímica $3+$ definido como una tinción completa e intensa del HER2 en la membrana de más de $10 \%$ de las células tumorales o FISH relación cociente HER2/CEP17 mayor de $2 .{ }^{39}$

\section{Enfermedad temprana}

El trastuzumab es un anticuerpo monoclonal dirigido contra el dominio extracelular del receptor de HER2, actúa inhibiendo su dimerización y activación, existen múltiples estudios en los que se demuestra su efectividad en el 
escenario adyuvante (HERA, FinHer, NSABP B-31, BCIRG006, N9831), especialmente en pacientes con tumores mayores de 1 centímetro con o sin ganglios positivos; de manera habitual el tratamiento con trastuzumab se administra de forma concomitante con quimioterapia basada en taxanos y se completa su administración durante un año. En el análisis de los estudios NSABP B-31 y NCCTG N9831 con una media de seguimiento de 8.4 años, la adición de trastuzumab a la quimioterapia produjo una mejoría de 37\% en SG (HR 0.63, 95\% IC, 0.54 a $0.73 ; \mathrm{p}<0.001)$ traducido como incremento de la SG a 10 años de 75.2 a 84\%. Esto fue asociado de igual forma a una mejoría en la supervivencia libre de enfermedad (SLE) de $40 \%$ (HR, 0.60; 95\% IC, 0.53 a 0.68; p < 0.001 ) traducido como incremento de la SLE a 10 años de 62.2 a $73.7 \% .{ }^{40,41} \mathrm{El}$ tratamiento con terapias anti-HER2 puede incrementar el riesgo de toxicidad cardiaca, principalmente asociada a una disminución asintomática de la fracción de eyección del ventrículo izquierdo, por lo que se recomienda monitorizar con ecocardiograma la función ventricular en estos pacientes de forma basal y posteriormente cada tres meses durante el periodo de tratamiento. ${ }^{42}$

El estudio HERA publicado en 2005 fue el primer estudio en el que se demostró el beneficio de trastuzumab adyuvante en pacientes con cáncer de mama HER2 positivo. Se incluyeron 5,102 pacientes en tres grupos, dos de ellos recibieron trastuzumab durante uno y dos años, respectivamente, y el grupo control (únicamente quimioterapia). La dosis utilizada fue de $8 \mathrm{mg} / \mathrm{kg}$ peso dosis inicial, seguida de $6 \mathrm{mg} / \mathrm{kg}$ dosis de mantenimiento cada tres semanas durante el tiempo establecido. En el seguimiento a 11 años se observó una mejoría estadísticamente significativa en la SLE (HR 0.76, 95\% IC 0.68-0.86) y una reducción del riesgo de muerte de $26 \%$ (HR $0.74,95 \%$ IC 0.64-0.86) cuando el trastuzumab fue administrado durante un año, comparado con el grupo de observación y sin encontrar diferencia al administrarlo durante dos años. ${ }^{43}$ Previamente, el beneficio de trastuzumab adyuvante había sido reportado en un metaanálisis publicado en 2012, en el que se observó una mejoría significativa en SG (HR 0.66, 95\% IC 0.57 a 0.77 , $\mathrm{p}<0.00001)$ y SLE (HR 0.60, 95\% IC 0.50 a
0.71, $\mathrm{p}<0.00001)$. Estableciéndose como el estándar de tratamiento en pacientes que no recibieron tratamiento neoadyuvante o que presentaron respuesta patológica completa. ${ }^{44}$

En pacientes que recibieron tratamiento neoadyuvante con quimioterapia más trastuzumab con o sin pertuzumab, la elección del tratamiento adyuvante dependerá de la respuesta que presenten al tratamiento neoadyuvante. En el estudio KATHERINE, publicado en 2019, se incluyeron pacientes con cáncer de mama que presentaran enfermedad invasiva residual en mama o axila en la pieza quirúrgica posterior a recibir tratamiento neoadyuvante basado en taxanos con o sin antraciclinas más trastuzumab, se comparó T-DM1 versus trastuzumab durante 14 ciclos en el escenario adyuvante, observando beneficio a favor del brazo de T-DM1 en supervivencia libre de enfermedad invasiva (SLEi) a tres años de 88.3 vs 77\% (HR $0.50,95 \%$ IC 0.39 a $0.64 ; p<0.001$ ), por lo que su uso fue aprobado para pacientes que recibieron tratamiento neoadyuvante con terapia anti-HER2 y no presentaron respuesta patológica completa. ${ }^{45}$

En pacientes en los que no se administró tratamiento neoadyuvante o bien presentaron respuesta patológica completa, se puede recomendar adyuvancia con trastuzumab \pm pertuzumab durante un año, el beneficio del doble bloqueo adyuvante fue establecido en el estudio APHINITY, en el cual se incluyeron pacientes con cáncer de mama HER2 positivo que presentaran ganglios positivos o factores de alto riesgo y se comparó quimioterapia más trastuzumab y pertuzumab versus quimioterapia más trastuzumab, identificando un beneficio en SLEi a tres años (94.1 vs 93.2\%), particularmente en el grupo con ganglios positivos 92 vs $90.2 \%$ (HR $0.77 ; 95 \%$ IC 0.62 a $0.96 ; p=0.02) .{ }^{46}$

La terapia extendida con inhibidor de HER2 fue valorado con neratinib; un inhibidor irreversible de tirosina-cinasa de EGFR. ExteNET fue un estudio en el que se incluyeron pacientes con cáncer de mama HER2 positivo en etapas iniciales que habían completado tratamiento adyuvante con trastuzumab, fueron aleatorizadas para recibir neratinib o placebo durante un año. El análisis del estudio demostró que neratinib comparado con placebo reducía el riesgo de recidiva de la enfermedad invasiva 
o de muerte 90.2 vs $87.7 \%$ (HR 0.73, 95\% IC 0.57 a $0.92, p=0.0083)$, particularmente en pacientes con tumores grandes y receptores hormonales positivos; sin embargo, a expensas de una mayor toxicidad grado 3 y 4 (40 vs $<$ $1 \%)$, principalmente gastrointestinal. ${ }^{47}$

\section{Enfermedad avanzada}

El uso de terapias anti-HER2 en el escenario avanzado ha sido determinante en la evolución natural de la enfermedad, mejorando el pronóstico de estos pacientes. El beneficio de trastuzumab en enfermedad avanzada como primera línea de tratamiento en combinación con quimioterapia quedó establecido en un estudio fase III en el que comparó la combinación de trastuzumab con paclitaxel o el esquema AC versus quimioterapia sola. Los resultados del estudio demostraron superioridad al agregar trastuzumab en respuesta global (50 vs 32\%), supervivencia libre de progresión (7.4 vs 4.6 meses) y supervivencia global (25.1 vs 20.3 meses). En un metaanálisis publicado en 2014 la adición de trastuzumab a la quimioterapia en pacientes con cáncer de mama avanzado HER2 positivo incrementó la SG (HR 0.82, 95\% IC 0.71 a 0.94, $\mathrm{p}=0.004)$ y SLE (HR $0.61,95 \%$ IC 0.54 a $0.70, p<0.00001) .48$

El tratamiento con doble bloqueo pertuzumab/trastuzumab como primera línea del cáncer de mama metastásico fue reportado en el estudio CLEOPATRA, en el que se valoró la combinación de trastuzumab, pertuzumab y docetaxel en comparación con docetaxel y trastuzumab; los resultados demostraron una SLP de 18.5 meses en el brazo de pertuzumab versus 12.4 meses en el brazo control (HR 0.62; $\mathrm{p}<0.001)$. En la SG la diferencia absoluta sin precedentes en este escenario fue de 16.3 meses (56.5 vs 40.8 meses) (HR 0.68; 95\% IC 0.58 a $0.80, p \leq 0.001)$. En el análisis final a ocho años de seguimiento $37 \%$ de los pacientes se encontraban vivos en el brazo de doble bloqueo. ${ }^{49}$

En pacientes refractarios a primera línea el uso de lapatinib, un inhibidor de la proliferación celular que bloquea el dominio intracelular al inhibir la acción de la tirosina-cinasa de HER1 y HER2, demostró beneficio cuando fue combinado con capecitabina en tiempo a la progresión (HR 0.49, 95\% IC 0.34 a 0.71, p <
0.001) con una media de tiempo a la progresión de 8.4 vs 4.4 meses, a expensas de una mayor toxicidad, principalmente gastrointestinal. ${ }^{50}$

El T-DM1 es un conjugado de anticuerpo y de fármaco que resulta de la combinación de trastuzumab y emtansina (también llamado DM1), la porción del anticuerpo bloquea la actividad del HER2 e incorpora la entrega intracelular del citotóxico emtansina, el cual es un potente inhibidor de microtúbulos. En la actualidad, el tratamiento en segunda línea en pacientes con cáncer de mama metastásico HER2 positivo es el uso de T-DM1. Lo anterior quedó demostrado en el estudio EMILIA que evaluó la utilidad del T-DM1 en pacientes previamente tratadas con trastuzumab y taxanos. Se aleatorizó a cualquiera de los dos brazos, T-DM1 versus la combinación de lapatinib + capecitabina. Los resultados demostraron un incremento en la SG de 30.9 vs 25.1 meses a favor de T-DM1 (HR $0.68, p<0.001)$. La respuesta objetiva también fue superior en el brazo experimental (43.6 vs $30.8 \%$ ). La principal toxicidad reportada fue trombocitopenia y elevación de transaminasas en el brazo de T-DM1. ${ }^{51}$

En la actualidad, debido a un mejor conocimiento biológico de estos tumores, se han desarrollado terapias dirigidas contra HER2 cada vez más sofisticadas, incluyendo nuevos anticuerpos con mayor afinidad, nuevos anticuerpos conjugados con fármacos, direccionamiento dual de epítopos a través de anticuerpos bioespecíficos, inhibidores de tirosina-cinasa más potentes y específicos, además de diferentes combinaciones. A continuación, hablaremos de las moléculas más recientemente aprobadas en pacientes con cáncer de mama HER2 positivo:

- Trastuzumab-deruxtecan (T-Dxd, DS8201): es un agente compuesto por un anticuerpo monoclonal humanizado anti-HER2 que tiene la misma secuencia de aminoácidos que el trastuzumab y un inhibidor de topoisomerasa I (deruxtecan, un derivado de exatecan 10 veces más potente que el metabolito activo del irinotecán). En el estudio fase 2 DESTINY-Breast 01, recientemente publicado que evaluó en beneficio de esta molécula en pacientes con cáncer de mama avanzado previamente tratados con T-DM1, el objetivo primario fue respuesta objetiva, 
la cual fue de $60.9 \%$ (95\% IC 54.3 a 68) con una media de duración de la respuesta de 14 meses y una mediana de supervivencia libre de progresión (SLPm) de16.4 meses. Los efectos secundarios más importantes fue neutropenia, anemia y náusea. ${ }^{52}$

- Margetuximab: es un anticuerpo monoclonal quimérico derivado del trastuzumab que se une al mismo epítopo con una mayor afinidad e incrementa la citotoxicidad mediada por células dependientes de anticuerpos. El estudio fase III SHOPIA comparó margetuximab más quimioterapia frente a trastuzumab más quimioterapia en pacientes con cáncer de mama avanzado que hubieran recibido por lo menos dos terapias anti-HER2 previas. El objetivo primario fue SLP y SG, en el segundo análisis interino se observó un beneficio en SLE de 5.8 vs 4.9 meses (HR 0.76, 95\% IC 0.59 a $0.98, p=0.033)$, sin beneficio en SG (HR $0.95,95 \%$ IC 0.69 a 1.31), mayor respuesta objetiva de 25.2 vs $13.7 \%$. La toxicidad fue muy similar en ambos grupos. Aún se encuentran pendientes los resultados finales de este estudio. ${ }^{53}$

- Neratinib/capecitabina: la combinación de inhibidor de tirosina cinasa y quimioterapia fue valorada en el estudio fase III NALA, en el cual se comparó neratinib más capecitabina vs lapatinib más capecitabina en pacientes previamente tratados. Reportando beneficio en SLP a 18 meses, 16 vs 7\% (HR 0.76, 95\% IC 0.63 a 0.93. $p=0$ 0.0059), sin diferencia estadísticamente significativa en SG. La toxicidad más frecuente fue diarrea. ${ }^{54}$

- Tucatinib: es un inhibidor de tirosina-cinasa, inhibe la fosforilación de HER2 y HER3. Su utilidad fue evaluada en el estudio fase III HER2CLIMB en pacientes con cáncer de mama localmente avanzado no resecable o metastásico que habían recibido terapia previa con trastuzumab, pertuzumab y T-DM1, el tratamiento incluía trastuzumab más capecitabina más tucatinib vs trastuzumab más capecitabina más placebo, el cual demostró reducción de $46 \%$ en el riesgo de progresión con una SLP a un año 33.1 vs $12.3 \%$ (HR 0.54, 95\% IC 0.42 a $0.51, \mathrm{p}<$ 0.001), también se observó reducción en el riesgo de muerte de $34 \%$, con una SG a dos años 44.9 vs $26.6 \%$ (HR 0.66, 95\% IC 0.50 a $0.88, p=0.005)$, mayor tasa de respuesta objetiva 40.6 vs $22.8 \%$, la toxicidad más común fue diarrea, elevación de transaminasas y eritrodisestesia palmo-plantar. ${ }^{55}$

De forma reciente, se ha puesto especial interés en el microambiente tumoral y las vías de señalización intracelular que juegan un papel fundamental en el desarrollo de resistencia a las terapias anti-HER2, por lo que se encuentran en desarrollo múltiples ensayos clínicos en los que se promueve el uso de combinaciones anticuerpo-inmunoterapia (inhibidores de PD-L1, CTLA4) anticuerpo-inhibidores específicos de señalización intracelular (RE, CDK4/6, PI3K, AKT, mTOR), de los cuales tendremos resultados en un futuro próximo. Aún quedan muchas preguntas por responder en el manejo óptimo del cáncer de mama HER2 positivo en los diferentes escenarios, por lo que es fundamental la identificación de biomarcadores predictivos para seleccionar de una mejor manera la secuencia y la población específica con la que se obtengan mejores resultados y favorecer el acceso de nuestros pacientes a estos tratamientos.

\section{ACTUALIDADES EN EL MANEJO DEL CÁNCER DE MAMATRIPLE NEGATIVO}

El subgrupo de pacientes con cáncer de mama, el cual carece de expresión de receptores hormonales y no sobreexpresa HER2 denominado triple negativo, presenta el pronóstico más sombrío con medianas de supervivencia global menores a 15 meses, ${ }^{56-58}$ esto como reflejo de limitadas opciones de tratamiento siendo la base de éste la quimioterapia sistémica.

La respuesta inmune está basada en un equilibrio entre activación e inhibición de las células que participan en ella. En el cáncer, este balance se encuentra alterado favoreciendo la inhibición del sistema inmune y con esto la progresión tumoral. PD1 (por sus siglas en inglés programmed cell death 1) es una proteína de membrana que se expresa en varias células del sistema inmune, incluyendo los linfocitos $\mathrm{T}, \mathrm{y}$ es activado por sus ligandos PD-L1 y PDL2. Al unirse con sus ligandos, PD1 atenúa la activa- 
ción linfocitaria y permite concluir la respuesta inmune. En cáncer de mama, la expresión de PD-L1 se encuentra incrementada en $20 \%$, con una mayor positividad asociada con características de mal pronóstico, como lo son mayor tamaño tumoral, alto grado tumoral, altos índices de proliferación y tumores triple negativos o con sobreexpresión de HER2. Por tanto, la reactivación de los linfocitos tumorales infiltrantes por los inhibidores de PD-L1 se planteó como una opción de tratamiento en las pacientes con cáncer de mama triple negativo. ${ }^{59}$

Atezolizumab es un anticuerpo monoclonal humanizado inhibidor selectivo de PD-L1, previene su unión con los receptores PD1 y B7-1 (proteína de superficie celular coestimuladora) y revierte la inhibición de las células tumorales sobre las células T. El tratamiento con quimioterapia incrementa la liberación de antígenos tumorales y con esto la respuesta inmune antitumoral. El ensayo clínico aleatorizado fase III (IMpassion 130) evaluó la eficacia del tratamiento con atezolizumab en pacientes con cáncer de mama triple negativo avanzado, vírgenes a tratamiento. El estudio aleatorizó en un radio 1:1 a 902 pacientes con estas características a recibir tratamiento con atezolizumab/nab-paclitaxel en comparación con placebo/nab-paclitaxel, resultando en una mediana de supervivencia libre de progresión de 7.2 meses con atezolizumab/ nab-paclitaxel en comparación con 5.5 meses con placebo/nab-paclitaxel $(\mathrm{HR} 0.8, \mathrm{p}=$ 0.002) y una mediana de supervivencia global de 25 meses en las pacientes con expresión de PD-L1 positivo (expresión de PD-L1 $\geq 1 \%$ en linfocitos tumorales infiltrantes evaluada mediante SP142, Ventana Medical Systems) en tratamiento con atezolizumab/nab-paclitaxel en comparación con 15.5 meses en pacientes tratadas con placebo/nab-paclitaxel (HR 0.62, 95\% IC 0.45-0.86). ${ }^{60}$

Pembrolizumab es otro anticuerpo monoclonal humanizado que bloquea PD1, inhibiendo la interacción entre PD1 y sus ligandos (PD-L1 y PDL2). El ensayo clínico fase III KEYNOTE-355 aleatorizó en un radio 2:1 a 847 pacientes con cáncer de mama avanzado triple negativo virgen a tratamiento a pembrolizumab más quimioterapia (a decisión del investigador: nab-paclitaxel, paclitaxel o gemcitabina más carboplatino) o placebo más quimioterapia, lo cual resultó en un mediana de supervivencia libre de progresión de 9.7 meses con pembrolizumab/quimioterapia en comparación de 5.6 meses en el grupo aleatorizado a placebo/quimioterapia (HR 0.65, $\mathrm{p}=$ 0.0012 ) en pacientes con un puntaje combinado positivo (CPS por sus siglas en inglés combined positive score) $\geq 10$ (número de células PDL1 positivas divididas entre el total de células tumorales $\times 100$, expresión de PD-L1 medida mediante IHC 22C3 pharmDx Assay). En las pacientes con PD-L1 CPS $<1$ la mediana de supervivencia libre de progresión no fue mejor con la adición de pembrolizumab al tratamiento (6.3 meses en el grupo de pembrolizumab/ quimioterapia en comparación con 6.2 meses en el grupo de placebo/quimioterapia. HR 1.08, $95 \%$ IC 0.77-1.53). ${ }^{61}$

\section{REFERENCIAS}

1. Bray F, Ferlay J, Soerjomataram I, Siegel RL, Torre LA, Jemal A. Global cancer statistics 2018: GLOBOCAN estimates of incidence and mortality worldwide for 36 cancers in 185 countries. CA Cancer J Clin 2018; 68: 394-424.

2. Bhargava R, Striebel J, Beriwal S, Flickinger J, Oniski A, Ahrendt $\mathrm{G}$ et al. Prevalence, morphologic features and proliferation indices of breast carcinoma molecular classes using immunohistochemical surrogate markers. Int J Clin Exp Pathol. 2009; 2: 444-455.

3. Cárdenas-Sánchez J, Erazo A, Arce-Salinas C, BargallóRocha JE, Bautista-Piña V, Cervantes-Sánchez G et al. Consenso Mexicano sobre diagnóstico y tratamiento del cáncer mamario. Octava revisión. Colima 2019. Gac Mex Oncol. 2019; 18: 141-231.

4. Howlader N, Altekruse SF, Li Cl, Chen VW, Clarke CA, Ries LAG et al. US Incidence of breast cancer subtypes defined by joint hormone receptor and HER2 status. J Natl Cancer Inst. 2014; 106 (5): dju055.

5. Wilcken N, Hornbuckle J, Ghersi D. Chemotherapy alone versus endocrine therapy alone for metastatic breast cancer. Cochrane Database Sys Rev. 2003; 2: CD002747.

6. Cardoso F, Senkus E, Costa A, Papadopoulos E, Aapro $\mathrm{M}$, André $\mathrm{F}$ et al. 4th ESO-ESMO International Consensus Guidelines for Advanced Breast Cancer (ABC 4). Ann Oncol. 2018; 29: 1634-1657.

7. Augusto TV, Correia-da-Silva G, Rodrigues CMP, Teixeira N, Amaral C. Acquired resistance to aromatase inhibitors: where we stand! Endocrine-Related Cancer. 2018; 25 (5): R283-R301.

8. Gibson L, Lawrence D, Dawson C, Bliss J. Aromatase inhibitors for treatment of advanced breast cancer in postmenopausal women. Cochrane Database Sys Rev. 2009; 4: CD003370.

9. Mouridsen H, Gershanovich M, Sun Y, Pérez-Carrión R, Boni C, Monnier A et al. Phase III study of letrozole versus tamoxifen as first-line therapy of advanced 
breast cancer in postmenopausal women: analysis of survival and update of efficacy from the international letrozole breast cancer group. J Clin Oncol. 2003; 21 (11): 2101-2109.

10. Paridaens RJ, Dirix LY, Beex LV, Nooij M, Cameron DA, Cufer T et al. Phase III study comparing exemestane with tamoxifen as first-line hormonal treatment of metastatic breast cancer in postmenopausal women: the European Organisation for Research and Treatment of Cancer Breast Cancer Cooperative Group. J Clin Oncol. 2008; 26: 4883-4890.

11. Ghosh D, Lo J, Egbuta C. Recent progress in the discovery of next generation inhibitors of aromatase from the structure-function perspective. J Med Chem. 2016; 59 (11): 5131-5148.

12. Chumsri S. Clinical utilities of aromatase inhibitors in breast cancer. Int J Womens Health. 2015; 7: 493-499.

13. Lonning PE. Lack of complete cross-resistance between different aromatase inhibitors; a real finding in search for an explanation? Eur J Cancer. 2009; 45: 527-535.

14. Bertelli G, Garrone O, Merlano M, Occelli M, Bertolotti $\mathrm{L}$, Castiglione F. Sequential treatment with exemestane and non-steroidal aromatase inhibitors in advanced breast cancer. Oncology. 2005; 69: 471-477.

15. Johnston SRD. Enhancing endocrine therapy for hormone receptor-positive advanced breast cancer: cotargeting signaling pathways. J Natl Cancer Inst. 2015; 107 (10): djv212.

16. Generali D, Fox SB, Brizzi MP, Allevi G, Bonardi S, Aguggini $S$ et al. Down-regulation of phosphatidylinositol 3'-kinase/AKT/molecular target of rapamycin metabolic pathway by primary letrozole-based therapy in human breast cancer. Clin Cancer Res. 2008; 14 (9): 2673-2680.

17. Miller TW, Balko JM, Arteaga CL. Phosphatidylinositol 3-kinase and antiestrogen resistance in breast cancer. J Clin Oncol. 2011; 29: 4452-4461.

18. Baselga J, Campone M, Piccart M, Burris III H, Rugo HS, Sahmoud T et al. Everolimus in postmenopausal hormone-receptor-positive advanced breast cancer. N Engl J Med. 2012; 366 (6): 520-529.

19. Bachelot T, Bourgier C, Cropet C, Ray-Coquard I, Ferrero JM, Freyer $\mathrm{G}$ et al. Randomized phase II trial of everolimus in combination with tamoxifen in patients with hormone receptor-positive, human epidermal growth factor receptor 2-negative metastatic breast cancer with prior exposure to aromatase inhibitors: a GINECO study. J Clin Oncol. 2012; 30 (22): 27182724.

20. Baselga J, Im SA, Iwata H, Cortés J, De Laurentiis $M$, Jiang Z et al. Buparlisib plus fulvestrant versus placebo plus fulvestrant in postmenopausal, hormone receptorpositive, HER2-negative, advanced breast cancer (BELLE-2): a randomised, double-blind, placebocontrolled, phase 3 trial. Lancet Oncol. 2017; 18 (7): 904-916.

21. André F, Ciruelos E, Rubovszky G, Campone M, Loibl S, Rugo HS. Alpelisib for PIK3CA-mutated, hormone receptor-positive advanced breast cancer. $\mathrm{N}$ Engl J Med. 2019; 380: 1929-1940.

22. Finn RS, Aleshin A, Slamon DJ. Targeting the cyclindependent kinases (CDK) 4/6 in estrogen receptor- positive breast cancers. Breast Can Res. 2016; 18: 17. 23. Fry DW, Harvey PJ, Keller PR, Elliott WL, Meade MA, Tracet $E$ et al. Specific inhibition of cyclin-dependent kinase 4/6 by PD 0332991 and associated antitumor activity in human tumor xenografts. Mol Cancer Ther. 2004; 3 (11): 1427-1438.

24. Marra A, Curigliano G. Are all cyclin-dependent kinases 4/6 inhibitors created equal? NPJ Breast Cancer. 2019; 5: 27.

25. Finn RS, Martin M, Rugo HS, Jones S, Im SA, Gelmon $\mathrm{K}$ et al. Palbociclib and letrozole in advanced breast cancer. N Engl J Med. 2016; 375 (20): 1925-1936.

26. Rugo HS, Finn RS, Diéras V, Ettl J, Lipatov O, Joy AA et al. Palbociclib plus letrozole as first-line therapy in estrogen receptor-positive/human epidermal growth factor receptor 2-negative advanced breast cancer with extended follow-up. Breast Cancer Res Treat. 2019; 174 (3): 719-729.

27. Hortobagyi GN, Stemmer SM, Burris HA, Yap YS, Sonke GS, Paluch-Shimon S et al. Ribociclib as firstline therapy for hr-positive, advanced breast cancer. N Engl J Med. 2016; 375 (18): 1738-1748.

28. Hortobagyi GN, Stemmer SM, Burris HA, Yap YS, Sonke GS, Paluch-Shimon $S$ et al. Updated results from MONALEESA-2, a phase III trial of first-line ribociclib plus letrozole versus placebo plus letrozole in hormone receptor-positive, HER2-negative advanced breast cancer. Ann Oncol. 2018; 29 (7): 1541-1547.

29. Goetz MP, Toi M, Campone M, Sohn J, Paluch-Shimon S, Huober J et al. MONARCH 3: abemaciclib as initial therapy for advanced breast cancer. J Clin Oncol. 2017; 35 (32): 3638-3646.

30. Tripathy D, Im SA, Colleoni M, Franke F, Bardia A, Harbeck $N$ et al. Ribociclib plus endocrine therapy for premenopausal women with hormone-receptorpositive, advanced breast cancer (MONALEESA-7): a randomised phase 3 trial. Lancet Oncol. 2018; 19: 904-919.

31. Im SA, Lu YS, Bardia A, Harbeck N, Colleoni M, Franke $\mathrm{F}$ et al. Overall survival with ribociclib plus endocrine therapy in breast cancer. N Engl J Med. 2019; 381 (4): 307-316.

32. Di Leo A, Jerusalem G, Petruzelka L, Torres R, Bondarenko IN, Khasanov R et al. Results of the CONFIRM phase III trial comparing fulvestrant $250 \mathrm{mg}$ with fulvestrant $500 \mathrm{mg}$ in postmenopausal women with estrogen receptor-positive advanced breast cancer. J Clin Oncol. 2010; 28 (30): 4594-4600.

33. Cristofanilli M, Turner NC, Bondarenko I, Ro J, Im SA, Masuda $\mathrm{N}$ et al. Fulvestrant plus palbociclib versus fulvestrant plus placebo for treatment of hormonereceptor-positive, HER2-negative metastatic breast cancer that progressed on previous endocrine therapy (PALOMA-3): final analysis of the multicentre, doubleblind, phase 3 randomised controlled trial. Lancet Oncol. 2016; 17 (4): 425-439.

34. Turner NC, Slamon DJ, Ro J, Bondarenko I, Im SA, Masuda $\mathrm{N}$ et al. Overall survival with palbociclib and fulvestrant in advanced breast cancer. N Engl J Med. 2018; 379: 1926-1936.

35. Slamon DJ, Neven P, Chia S, Fasching PA, De Laurentiis $M, I m$ SA et al. Phase III randomized study of ribociclib and fulvestrant in hormone receptor-positive, human 
epidermal growth factor receptor 2-negative advanced breast cancer: MONALEESA-3. J Clin Oncol. 2018; 36 (24): 2465-2472.

36. Sledge GW, Toi M, Neven P, Sohn JM, Inoue K, Pivot X et al. MONARCH 2: abemaciclib in combination with fulvestrant in women with HR+/HER2-avanced breast cancer who had progressed while receiving endocrine therapy. J Clin Oncol. 2017; 35 (25): 2875-2884.

37. Loibl S, Gianni L. HER2-positive breast cancer. Lancet. 2017; 389 (10087): 2415-2429.

38. Yarden Y, Sliwkowski MX. Untangling the ErbB signalling network. Nat Rev Mol Cell Biol. 2001; 2 (2): 127-37.

39. Wolff AC, Hammond ME, Hicks DG, Dowsett M, McShane LM, Allison KH et al. Recommendations for human epidermal growth factor receptor 2 testing in breast cancer: American Society of Clinical Oncology/College of American Pathologists clinical practice guideline update. J Clin Oncol. 2013; 31 (31): 3997 4013.

40. Denduluri N, Chavez-MacGregor M, Telli ML, Eisen A, Graff SL, Hassett MJ et al. Selection of optimal adjuvant chemotherapy and targeted therapy for early breast cancer: ASCO clinical practice guideline focused update. J Clin Oncol. 2018; 36 (23): 2433-2443.

41. Perez EA, Romond EH, Suman VJ, Jeong JH, Sledge G, Geyer CE Jr et al. Trastuzumab plus adjuvant chemotherapy for human epidermal growth factor receptor 2-positive breast cancer: planned joint analysis of overall survival from NSABP B-31 and NCCTG N9831. J Clin Oncol. 2014; 32 (33): 3744-3752.

42. Keefe DL. Trastuzumab-associated cardiotoxicity. Cancer. 2002; 95 (7): 1592-600.

43. Cameron D, Piccart-Gebhart MJ, Gelber RD, Procter M, Goldhirsch A, de Azambuja E et al. 11 years' follow-up of trastuzumab after adjuvant chemotherapy in HER2-positive early breast cancer: final analysis of the HERceptin adjuvant (HERA) trial. Lancet. 2017; 389 (10075): 1195-1205.

44. Moja L, Tagliabue L, Balduzzi S, Parmelli E, Pistotti V, Guarneri $\vee$ et al. Trastuzumab containing regimens for early breast cancer. Cochrane Database Syst Rev. 2012; 2012 (4): CD006243.

45. Von Minckwitz G, Huang CS, Mano MS, Loibl S, Mamounas EP, Untch $M$ et al. Trastuzumab emtansine for residual invasive HER2-positive breast cancer. N Engl J Med. 2019; 380 (7): 617-628.

46. Von Minckwitz G, Procter M, de Azambuja E, Zardavas $D$, Benyunes $M$, Viale $G$ et al. Adjuvant pertuzumab and trastuzumab in early HER2-positive breast cancer. N Engl J Med. 2017; 377 (2): 122-131.

47. Martin M, Holmes FA, Ejlertsen B, Delaloge S, Moy $\mathrm{B}$, Iwata $\mathrm{H}$ et al. Neratinib after trastuzumab-based adjuvant therapy in HER2-positive breast cancer (ExteNET): 5-year analysis of a randomised, double-blind placebo-controlled, phase 3 trial. Lancet Oncol. 2017; 18 (12): 1688-1700.

48. Balduzzi S, Mantarro S, Guarneri V, Tagliabue L, Pistotti $V$, Moja $L$ et al. Trastuzumab-containing regimens for metastatic breast cancer. Cochrane Database Syst Rev. 2014; 2014 (6): CD006242.

49. Swain SM, Baselga J, Kim SB, Ro J, Semiglazov V, Campone $\mathrm{M}$ et al. Pertuzumab, trastuzumab, and docetaxel in HER2-positive metastatic breast cancer. N Engl J Med. 2015; 372 (8): 724-734.

50. Geyer CE, Forster J, Lindquist D, Chan S, Romieu CG, Pienkowski $T$ et al. Lapatinib plus capecitabine for HER2-positive advanced breast cancer. N Engl J Med. 2006; 355 (26): 2733-2743.

51. Verma S, Miles D, Gianni L, Krop IE, Welslau M, Baselga J et al. Trastuzumab emtansine for HER2positive advanced breast cancer. N Engl J Med. 2012; 367 (19): 1783-1791.

52. Modi S, Saura C, Yamashita T, Park YH, Kim SB, Tamura $\mathrm{K}$ et al. Trastuzumab deruxtecan in previously treated HER2-positive breast cancer. N Engl J Med. 2020; 382 (7): 610-621.

53. Rugo HS, Im SA, Cardoso F et al. Abstract GS102: phase 3 SOPHIA study of margetuximab + chemotherapy vs trastuzumab + chemotherapy in patients with HER2 + metastatic breast cancer after prior anti-HER2 therapies: second interim overall survival analysis. Cancer Res. 2020; 80 (4 Suppl): GS1-02.

54. Saura C, Oliveira M, Feng YH, Dai MS, Chen SW, Hurvitz SA et al. Neratinib plus capecitabine versus lapatinib plus capecitabine in HER2-positive metastatic breast cancer previously treated with $\geq 2$ HER2directed regimens: phase III NALA trial. J Clin Oncol. 2020; 38 (27): 3138-3149.

55. Murthy RK, Loi S, Okines A, Paplomata E, Hamilton E, Hurvitz SA et al. Tucatinib, trastuzumab, and capecitabine for HER2-positive metastatic breast cancer. N Engl J Med. 2020; 382 (7): 597-609.

56. Brok WD, Speers CH, Gondara L, Baxter E, Tyldesley SK, Lohrisch CA. Survival with metastatic breast cancer based on initial presentation, de novo versus relapsed. Breast Can Res Treatment. 2017; 161: 549-556.

57. Gobbini E, Ezzalfani M, Dieras V, Bachelot T, Brain E, Debled $\mathrm{M}$ et al. Time trends of overall survival among metastatic breast cancer patients in the real-life ESME cohort. Eur J Cancer. 2018; 96: 17-24.

58. Deluche E, Antoine A, Bachelot T, Lardy-Cleaud A, Dieras V, Brain E et al. Contemporary outcomes of metastatic breast cancer among 22,000 women from the multicentre ESME cohort 2008-2016. Eur J Cancer. 2020; 129: 60-70.

59. Sabatier R, Finetti P, Mamessier E, Adelaide J, Chaffanet $\mathrm{M}$, Raza $\mathrm{H}$ et al. Prognostic and predictive value of PDL1 expression in breast cancer. Oncotarget. 2014; 6 (7): 5449-5464.

60. Schmid P, Adams S, Rugo HS, Schneeweiss A, Barrios $\mathrm{CH}$, Iwata $\mathrm{H}$ et al. Atezolizumab and nab-paclitaxel in advanced triple-negative breast cancer. N Engl J Med. 2018; 379: 2108-2121.

61. Cortes J, Cescon DW, Rugo HS, Nowecki Z, Im SA, Yusof $M$ et al. Pembrolizumab plus chemotherapy versus placebo plus chemotherapy for previously untreated locally recurrent inoperable o metastatic triple-negative breast cancer (KEYNOTE-355): a randomised, placebo-controlled, double-blind, phase 3 clinical trial. Lancet. 2020; 396: 1817 1828. 